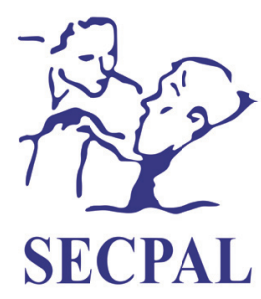

Medicina Paliativa

www.medicinapaliativa.es

ORIGINAL

\title{
Cuidados en el final de la vida y estudiantes de Ciencias de la Salud: ¿binomio bien avenido?
}

\author{
Sagrario Pérez de la Cruz*
}

Departamento de Enfermería, Fisioterapia y Medicina, Universidad de Almería, España

Recibido el 16 de enero de 2018

Aceptado el 25 de marzo de 2018

\section{PALABRAS CLAVE \\ Estudiantes, final de \\ la vida, formación.}

\begin{abstract}
Resumen
Objetivo: Conocer el grado de capacitación, el grado de seguridad autopercibida y las preferencias que tienen los estudiantes de Ciencias de la Salud en relación con la asistencia a enfermos terminales y no terminales.
\end{abstract}

Método: Estudio descriptivo, transversal y multicéntrico. La muestra estuvo formada por tres grupos: estudiantes de Medicina, de Enfermería y de Fisioterapia. Los criterios de inclusión de estudiantes en esta muestra fueron: ser mayor de 18 años, estar matriculados en tercer año de carrera y firmar el consentimiento informado de participación en este estudio. Duración: entre noviembre de 2016 y marzo de 2017. Variables evaluadas: preparación asistencial y preparación emocional para cuidar enfermos terminales y no terminales, y predilección laboral.

Resultados: De los 410 estudiantes que participaron en la muestra, la preparación autopercibida de los estudiantes para cuidar enfermos terminales, desde un punto de vista asistencial, se puede considerar "regular" (media 2,15; desviación estándar 0,756), al igual que para atender de manera emocional a estos pacientes (media 2,19; desviación estándar 0,827). Sin embargo, para cuidar y atender emocionalmente a enfermos no terminales, la puntuación obtenida es superior (2,99 y 3,16 de media, respectivamente). En cuanto a la preferencia laboral, la alternativa de trabajo con enfermos terminales, ya sea enfermos de cáncer terminales o geriátricos, es la opción menos aceptada por los futuros profesionales de la salud.

Conclusión: Se observa poca predilección por los cuidados y tratamiento en el final de la vida, así como poca preparación de los estudiantes de Ciencias de la Salud para trabajar con estos pacientes.

\footnotetext{
*Autor para correspondencia:

Sagrario Pérez de la Cruz

Facultad de Ciencias de la Salud. Despacho 2050, Universidad de Almería. Carretera de Sacramento s/n. 04120 La Cañada de San Urbano. Almería.

Correo electrónico: spd205@ual.es
}

DOI: 10.20986/medpal.2019.1043/2019

1134-248X/ ( 2019 Sociedad Española de Cuidados Paliativos. Publicado por Inspira Network. Todos los derechos reservados. 


\section{KEYWORDS}

Students, end of life, training.

\begin{abstract}
Aim: To know the interest shown by Heath Sciences students in relation to working with terminal and nonterminal patients, and to working in certains units with these patients.

Method: A descriptive, cross-sectional, multicenter study with three groups: Medicine, Nursing, and Physiotherapy students. Inclusion criteria for students in this sample included: age over 18 years, third year of school, and signing an informed consent for participation in the study between November 2016 and March 2017. Assessed variables included: technical and emotional preparation for caring for terminal or nonterminal patients, and occupational predilection.

Results: Among the 410 students in the sample self-perceived preparation for caring for terminal patients may be considered "moderate" both from a healthcare perspective, (mean, 2.15; standard deviation, 0.756) and an emotional standpoint (mean, 2.19; standard deviation, 0.827). However, for caring and emotionally approaching nonterminal patients a higher score was obtained (mean, 2.99 and 3.16, respectively). As regards job preferences, working with end-stage patients, whether terminal cancer or geriatric individuals, was the less desirable option for future healthcare professionals.
\end{abstract}

Conclusion: Predilection is low for end-of-life care and management, and Health Sciences students are poorly prepared to work with these patients.

Pérez de la Cruz S. Cuidados en el final de la vida y estudiantes de Ciencias de la Salud: ¿binomio bien avenido? Med Paliat. $2019 ; 26(2): 97-103$.

\section{Introducción}

La muerte ha sido siempre, y es, para el ser humano, un tema de profundas reflexiones y meditaciones, ya sea desde la perspectiva filosófica, religiosa o científica. Sin embargo, en las sociedades industrializadas avanzadas cada vez es más difícil convivir con la idea de la muerte o aceptarla¹. El concepto y las actitudes hacia la muerte han venido sufriendo una "evolución" en los últimos siglos y, sobre todo, en las últimas décadas, de modo que su mismo significado semántico se ha visto modificado ${ }^{2}$. Los avances de la Medicina y otras ciencias afines permiten la prolongación de la vida o el mantenimiento de funciones vitales hasta límites insospechados. Sumando todo ello al envejecimiento de la población y al consiguiente incremento de personas con enfermedades crónicas, hace que haya aumentado el número de situaciones que llegan a situación terminal, con pronóstico vital limitado y un intenso sufrimiento personal y familiar, con frecuencia en un contexto de atención sanitaria altamente tecnificada3,4.

Cada vez se pone más en evidencia la importancia de la actitud de los profesionales sanitarios frente al proceso de la muerte y el dolor ${ }^{5}$. Por un lado está la calidad de su relación con el enfermo en la elaboración del duelo, tanto del propio enfermo como de la familia próxima, así como a la hora de recibir las noticias, o en los instantes de muerte y duelo. Este supuesto se adjudica a los profesionales de la Enfermería (colectivo que tiene mayor participación en el proceso de la defunción). Pero en el caso de los médicos, el tema de la muerte es sentido de otra manera, con otra perspectiva. Se supone que es el profesional mejor entrenado en su manejo ${ }^{6}$. No obstante, no siempre es así, y con frecuencia se refleja en la poca atención directa al paciente.

Los estudiantes de Medicina y Enfermería deberían estar entrenados, una vez finalizada su formación, en el manejo del proceso de la muerte de los pacientes a su cargo, que debería tener cabida en los planes de estudios. Es evidente que para prestar la mejor atención posible al paciente en situación de enfermedad avanzada y terminal y a sus familiares es necesaria una formación específica en la fase del duelo, muerte, enfermedad terminal y cuidados paliativos, tanto en la formación pregrado como durante el desempeño profesional $^{7,8}$.

También, en los últimos años se ha incrementado la formación en cuidados paliativos, incluyéndose en el currículum formativo de carreras sanitarias. En muchas universidades españolas imparten una asignatura o módulos sobre cuidados paliativos; sin embargo, estos no son obligatorios. Tampoco hay unanimidad con respecto al contenido y la cantidad de horas dedicadas a este tipo de capacitación. Además, los estudiantes no tienen la misma oportunidad de recibir capacitación básica en cuidados paliativos en todas las facultades ${ }^{9-13}$.

Aunque la situación está cambiando, en la actualidad el modelo de enseñanza y ubicación clínica que se enseña en las universidades españolas dedica un tiempo de estudio insuficiente a las cuestiones relacionadas con la muerte. Esta falta de capacitación específica en cuidados paliativos es, en gran parte, responsable de los sentimientos de inseguridad y evitación mostrados por algunos profesionales hacia el final de la vida de sus pacientes. Esto interfiere en las emociones tanto del profesional como de la familia, así como de los pacientes, lo que significa que estas emociones se convierten en un obstáculo para la toma de decisiones ${ }^{14,15}$.

El objetivo principal del estudio es conocer la capacitación y preparación que tienen los estudiantes de Ciencias de la Salud (Medicina, Enfermería y Fisioterapia) en relación con la asistencia a enfermos terminales y no terminales, y evaluar el interés y preferencias que muestran respecto a 
desarrollar su profesión en determinados servicios (geriatría, unidades de vigilancia intensiva, enfermedades neurodegenerativas y cuidados paliativos) así como su grado de seguridad autopercibida en el manejo de pacientes en situación de enfermedad terminal.

\section{Material y métodos}

El estudio se ajusta a un diseño descriptivo, transversal y multicéntrico.

\section{Muestra del estudio}

La muestra estuvo formada por tres grupos: estudiantes de Medicina, de Enfermería y de Fisioterapia. Fueron seleccionados a lo largo del curso 2016-2017 (concretamente, entre noviembre de 2016 y marzo de 2017).

La muestra total fue de 410 alumnos, repartidos de la manera que se refleja en la Tabla I. Pertenecían a seis facultades de Ciencias de Salud repartidas por el territorio español.

Los criterios de selección de inclusión de estudiantes en esta muestra fueron: ser mayor de 18 años, estar matriculados en tercer año de carrera (de las tres titulaciones propuestas) y firmar el consentimiento informado de participación en este estudio. La participación fue voluntaria y no recibieron ninguna retribución por su colaboración (muestreo no aleatorizado). Se excluyeron aquellos estudiantes que no cumplían dichos requisitos.

Es importante poder contar con la opinión de tres titulaciones de Ciencias de la Salud, las cuales, dentro de su desarrollo laboral, tendrán contacto y relación con pacientes en la última etapa de su vida y próximos a la muerte.

\section{Instrumentos}

Los instrumentos empleados para medir y cuantificar los resultados de la medición de las variables en este estudio fueron:

- Variable Preparación asistencial y preparación emocional que manifiesta el sujeto para cuidar pacientes con enfermedad terminal ${ }^{16}$. Se pregunta a los participantes si se sienten preparados o capaces, entre otros aspectos, de controlar el dolor, comunicarse y tratar los diversos síntomas que van apareciendo en el proceso de morir. Las respuestas podían ir desde "Nada" (con una puntua-

Tabla I. Distribución de los alumnos participantes en el estudio según titulación y sexo

\begin{tabular}{lccc}
\hline Titulación & $\begin{array}{c}\text { Número } \\
\text { total (\%) }\end{array}$ & Varones (\%) & Mujeres (\%) \\
\hline Enfermería & $143(35,03)$ & $30(20,8)$ & $113(79,2)$ \\
Fisioterapia & $159(38,7)$ & $81(59,9)$ & $78(49,1)$ \\
Medicina & $108(26,27)$ & $21(19,4)$ & $87(80,6)$ \\
\hline
\end{tabular}

ción de 1), "Poco" (con una puntuación de 2), “Regular" (puntuación de 3), "Bastante” (que puntuaba 4) y "Mucho" (con una puntuación de 5).

- Variable Preparación emocional y asistencial para cuidar enfermos de patología general ${ }^{16}$. Con esta cuestión y la anterior se pretende conocer si la sensación de preparación emocional y técnica es la misma a la hora de cuidar personas enfermas, pero que no necesariamente estén al final de su vida. La puntuación empleada es la descrita anteriormente.

- Variable Preferencias profesionales en el campo del cuidado del enfermo. Para averiguar las preferencias de los futuros profesionales de la salud una vez concluidos sus estudios, se elaboró un cuestionario en el que se recogen distintos ámbitos de trabajo. Al alumno se le preguntó su deseo o no de trabajar en determinados campos de la salud. La puntuación va desde 1 ("No lo desearía en absoluto”) hasta 7 (“Lo desearía muchísimo”).

\section{Procedimiento}

Partiendo de una muestra geográficamente dispersa en el territorio nacional (ya que son diferentes facultades, para así poder contar con muestra diversa), se contactó con los profesores responsables de asignaturas que se estaban impartiendo en ese periodo lectivo. Una vez recibida respuesta por parte de los responsables de las asignaturas de las distintas titulaciones, nos citamos en día, hora y lugar para poder desplazarnos y facilitar los cuestionarios de manera presencial.

Teniendo en cuenta que el dosier suponía un periodo de tiempo no inferior a 15 minutos, los responsables de las asignaturas cedieron la mitad de su hora de clase (aproximadamente 30 minutos) para explicar a los alumnos participantes en qué consistía el estudio y aclarar cualquier duda que pudiera surgir a la hora de cumplimentar el mismo. Se repartieron los cuestionarios autoadministrados y anónimos, y se recogieron una vez transcurridos 30 minutos. Los participantes tuvieron tiempo suficiente para contestar a las preguntas con tranquilidad.

\section{Análisis estadístico}

Los datos fueron tabulados en el Software IBM ${ }^{\circledR}$ SPSS ${ }^{\circledR}$ Statistics V21.0.0. Para el análisis, previamente se les asignó un código numérico con el fin de preservar el anonimato de los participantes.

Se analizaron las variables sociodemográficas o de caracterización. Las variables estudiadas fueron: sexo, edad, estudios y población de origen. Se describieron frecuencias, porcentajes, medias y desviaciones típicas.

Para poder comparar las puntuaciones obtenidas entre las titulaciones y sexos, y concluir si tales diferencias eran estadísticamente significativas, se emplearon pruebas no paramétricas, debido a que existía una distribución normal. Se utilizó el test de Mann-Whitney para examinar las diferencias entre grupos.

Las pruebas no paramétricas empleadas fueron el test de Wilcoxon-Mann-Whitney (para dos muestras independientes), el test de Kruskal-Wallis (para k-muestras indepen- 
dientes) y el test de $\mathrm{x}$-cuadrado (para dos muestras cualitativas independientes). En todos los casos los resultados presentados se ciñen a un intervalo de confianza del $95 \%$.

\section{Resultados}

De 410 alumnos que participaron en la muestra, la edad media fue 23,3 años, siendo la distribución por titulación: Enfermería (24,3 años), Fisioterapia (23,7 años) y Medicina (21,9 años). Todos los participantes eran alumnos matriculados en tercer año de su respectivo grado universitario.

Respecto al lugar de residencia habitual, el 28,3\% de ellos vive en municipios con población inferior a 5.000 habitantes, el $43,8 \%$ vive en poblaciones de entre 5.000 a 100.000 habitantes y el resto $(27,9 \%)$ lo hace en ciudades con población superior a 100.000 habitantes.

En la Tabla II y Figuras 1 y 2 se muestran los datos obtenidos para las cuestiones referentes a la capacidad asis- tencial y emocional para cuidar enfermos terminales y no terminales.

\section{Predilección laboral}

Para los tres colectivos encuestados, el área de enfermos geriátricos terminales no es un campo de trabajo atractivo, mostrando el colectivo de Enfermería mayor rechazo a trabajar en esta área, mientras que el de Medicina se muestra indiferente. Fisioterapia es una profesión que no muestra mucha afinidad. En líneas generales se puede afirmar que no es un campo de trabajo deseado por los futuros profesionales encuestados. Aquí se ven diferencias estadísticamente significativas entre todas las titulaciones $(p=0,000$; $x$-cuadrado 19,148; GL con valor 2).

Otra de las opciones en la que los resultados han mostrado diferencias significativas es en la preferencia de trabajo con pacientes enfermos en unidades de cuidados intensivos

Tabla II. Capacidad asistencial y emocional para cuidar enfermos terminales y no terminales

\begin{tabular}{|c|c|c|c|c|c|}
\hline \multicolumn{2}{|c|}{ Alumnos } & $\begin{array}{l}\text { ¿Se siente usted } \\
\text { preparado desde un } \\
\text { punto de vista } \\
\text { asistencial para cuidar } \\
\text { enfermos terminales? }\end{array}$ & $\begin{array}{l}\text { ¿Se siente usted } \\
\text { preparado desde un } \\
\text { punto de vista } \\
\text { asistencial para cuidar } \\
\text { enfermos NO } \\
\text { terminales? }\end{array}$ & $\begin{array}{c}\text { ¿Se siente usted } \\
\text { preparado desde un } \\
\text { punto de vista } \\
\text { emocional para cuidar } \\
\text { enfermos terminales? }\end{array}$ & $\begin{array}{l}\text { ¿Se siente usted } \\
\text { preparado desde un } \\
\text { punto de vista } \\
\text { emocional para cuidar } \\
\text { enfermos NO } \\
\text { terminales? }\end{array}$ \\
\hline \multirow{2}{*}{ Enfermería } & Media & 2,25 & 2,92 & 2,17 & 2,92 \\
\hline & SD & 0,668 & 0,498 & 0,753 & 0,613 \\
\hline \multirow{2}{*}{ Fisioterapia } & Media & 2,00 & 2,98 & 2,09 & 3,30 \\
\hline & SD & 0,784 & 0,635 & 0,815 & 0,668 \\
\hline \multirow{2}{*}{ Medicina } & Media & 2,25 & 3,08 & 2,36 & 3,28 \\
\hline & SD & 0,806 & 0,554 & 0,931 & 0,566 \\
\hline \multirow{2}{*}{ Total } & Media & 2,15 & 2,99 & 2,19 & 3,16 \\
\hline & SD & 0,756 & 0,569 & 0,827 & 0,644 \\
\hline
\end{tabular}

SD: desviación típica.

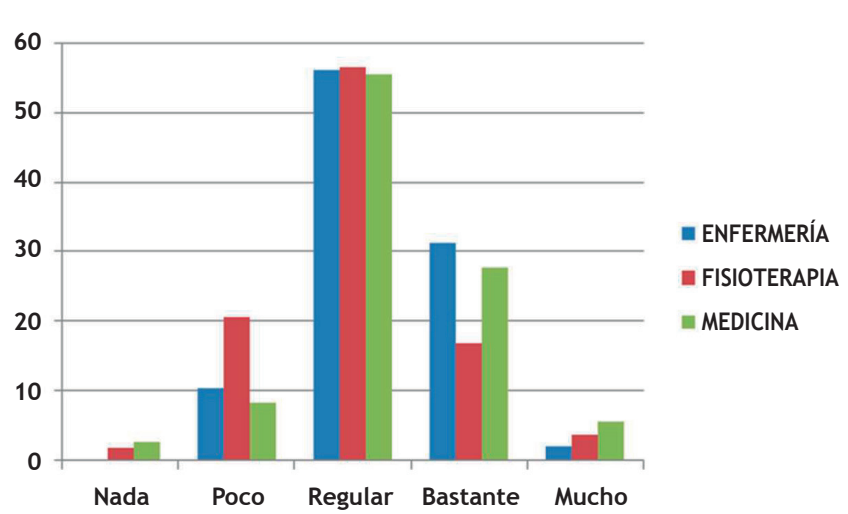

Figura 1. Distribución de los porcentajes de estudiantes que hacen referencia a la preparación asistencial para cuidar enfermos terminales.

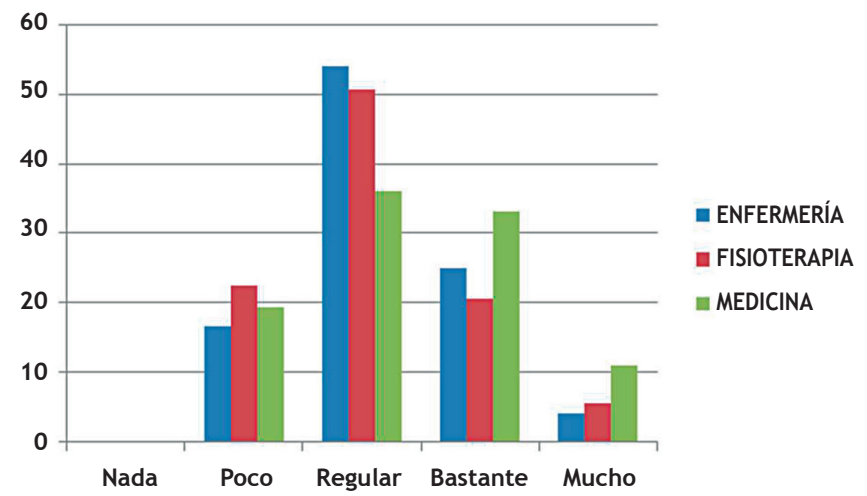

Figura 2. Distribución de los porcentajes de estudiantes que hacen referencia a la preparación emocional para cuidar enfermos terminales. 
$(p=0,000)$ y con grandes prematuros $(p=0,011)$. En este último caso los futuros profesionales de Enfermería han mostrado mayor preferencia que las otras dos titulaciones implicadas en el estudio.

En la Tabla III se muestran los resultados para la cuestión de la predilección laboral.

\section{Discusión}

Los profesionales de la salud en España muestran dificultades de tipo cultural, social y religioso para afrontar la muerte y asistir y acompañar a los enfermos terminales y sus familias en el proceso $8,17-21$.

En la actualidad, la muerte es un tema tabú en la cultura occidental (a diferencia de otras culturas, donde la muerte es vista con más naturalidad), ya que se nos vende la omnipotencia de los medios técnicos y tecnológicos para poder curar en el ámbito de la medicina y, en general, de la salud, lo que genera inseguridad, desagrado y ansiedad ante la "victoria" de la muerte o enfermedad terminal ${ }^{17-21}$. Además, la actitud ante la muerte del profesional se hace extensible al hecho de cómo poder responder a las solicitudes de la familia del moribundo ${ }^{18-22}$.

Trabajos como los defendidos por Benbunan-Bentata y cols. ${ }^{5}$ inciden en otro aspecto también muy importante en ese momento de transición (vida-muerte), como es la presencia, implicación, afectos y efectos de las emociones mostradas por los profesionales de la salud (en nuestro caso, futuros profesionales sanitarios) y cómo afrontan la ansiedad y estrés que se deriva de esa situación.

Para superar con éxito toda esa situación estresante es necesario llevar a cabo un programa de evaluación, habilidades de comunicación e información y soporte psicosocial, así como dar importancia a las cuestiones bioéticas y de trabajo interdisciplinar entre los profesionales, y más especialmente en las profesiones de médicos y enfermeras, por encontrarse más a menudo en situaciones de cercanía con la muerte. Los estudiantes y profesionales no deben estar únicamente formados en conocimientos técnico-científicos, sino que tienen que comportarse como personas sensibles al sufrimiento humano. Por eso, se define la relación sanitariopaciente como una relación eminentemente humanista. Se debe fomentar un ambiente en el que los valores, costumbres y creencias del individuo sean respetados, ayudando al enfermo a mantener, desarrollar o adquirir autonomía personal, respeto y determinación en su propias decisiones, siempre guiándose por criterios profesionales y buscando el bien del paciente ${ }^{5}$.

Una actitud de empatía de los profesionales con el sufrimiento resulta inexcusable para el acompañamiento al final de la vida. Asimismo, hay que individualizar las intervenciones y reconocer que la variabilidad emocional interpersonal, en la vivencia ante la muerte constituye el entramado de la atención paliativa ${ }^{23,24}$.

Es de suponer, teóricamente, que si los alumnos no se sienten preparados desde el punto de vista emocional, menos lo estarán desde el asistencial, ya sea para cuidar enfermos terminales como no terminales.

Esta afirmación se demostró en los estudios de Colell ${ }^{16}$, que obtuvieron puntuaciones mayores en la preparación emocional que en la asistencial, expresando mayores dificultades en la interrelación y comunicación con enfermos terminales, así como con el entorno familiar más cerca$n^{25}$. Este hecho va en consonancia con los resultados que hemos obtenido en nuestra muestra, sin distinción entre profesionales, ya que en nuestro estudio la preparación emocional supera igualmente a la asistencial. Esta similitud puede deberse a la formación que reciben en los estudios de pregrado, en los cursos iniciales, o bien a experiencias personales $\mathrm{y} / \mathrm{o}$ de las prácticas clínicas.

Por otra parte, cabe destacar también la baja puntuación obtenida respecto a la sensación de preparación académica y asistencial para cuidar enfermos terminales, como ocurre igualmente en otros estudios similares ${ }^{26-28}$. También se ha evidenciado que ciertas asignaturas no son ofertadas de manera obligatoria, de forma que los cuidados paliativos no son una opción muy buscada por los estudiantes, conside-

Tabla III. Predilección que muestran los estudiantes de la muestra para desarrollar su labor profesional en distintos servicios sanitarios con pacientes próximos a la muerte

\begin{tabular}{|c|c|c|c|c|c|c|}
\hline \multicolumn{2}{|c|}{ Alumnos } & $\begin{array}{c}\text { Trabajar con } \\
\text { enfermos en un } \\
\text { hospital de } \\
\text { agudos }\end{array}$ & $\begin{array}{l}\text { Trabajar con } \\
\text { enfermos } \\
\text { terminales de } \\
\text { cáncer }\end{array}$ & $\begin{array}{l}\text { Trabajar con } \\
\text { enfermos } \\
\text { terminales } \\
\text { geriátricos }\end{array}$ & $\begin{array}{c}\text { Trabajar con } \\
\text { enfermos } \\
\text { ingresados en la } \\
\text { UCI }\end{array}$ & $\begin{array}{c}\text { Trabajar con } \\
\text { pacientes con } \\
\text { enfermedades } \\
\text { neurodegenerativas }\end{array}$ \\
\hline \multirow{2}{*}{ Enfermería } & Media & 4,94 & 4,58 & 3,67 & 5,13 & 4,27 \\
\hline & $\mathrm{DE}$ & 1,295 & 1,686 & 1,642 & 1,378 & 1,469 \\
\hline \multirow{2}{*}{ Fisioterapia } & Media & 4,87 & 3,09 & 3,30 & 3,75 & 4,40 \\
\hline & $\mathrm{DE}$ & 1,618 & 1,390 & 1,295 & 1,686 & 1,511 \\
\hline \multirow{2}{*}{ Medicina } & Media & 5,11 & 3,97 & 3,72 & 4,78 & 3,97 \\
\hline & $\mathrm{DE}$ & 1,430 & 1,748 & 1,504 & 1,884 & 1,483 \\
\hline \multirow{2}{*}{ Total } & Media & 4,96 & 3,85 & 3,54 & 4,50 & 4,24 \\
\hline & $\mathrm{DE}$ & 1,455 & 1,710 & 1,480 & 1,741 & 1,488 \\
\hline
\end{tabular}

UCI: unidad de cuidados intensivos; DE: desviación estándar. 
rando "muy triste y deprimente" trabajar con este tipo de pacientes. No consideran que necesiten cuidados especiales ni una preparación específica, diferente al resto de enfermos de un centro sanitario ${ }^{4}$.

Puede que parte de esta concepción provenga de los propios centros universitarios, que deberían profundizar en mostrar la realidad a la que se van a enfrentar los estudiantes, ya que los procesos de enfermedad suelen darse en una etapa final de la vida y la esperanza de vida es elevada. Se debería concienciar a los estudiantes sobre el hecho de que sus usuarios pueden ser personas cuya única salida a su afección es la muerte, y no siempre la recuperación/ rehabilitación total y plena.

La necesidad de mejorar la formación de los futuros profesionales ${ }^{26,27}$ (o de los propios profesionales en ejercicio) en todo lo relacionado con la enfermedad terminal (dándose más frecuentemente en enfermedades crónicas y degenerativas avanzadas) es un objetivo que las Administraciones sanitarias se han planteado. Sin embargo, siguen ignorándose o evitándose referencias al factor "personal" de los profesionales que trabajan con estos enfermos y las familias, tanto en el nivel asistencial como en las propuestas de mejora-acompañamiento.

Otra de las cuestiones que queríamos conocer eran las preferencias laborales que mostraban los futuros profesionales de la salud. La elección preferida por los estudiantes de Enfermería es la atención de pacientes pediátricos, grandes prematuros y unidades de cuidados intensivos. Los servicios menos elegidos fueron las unidades de enfermos terminales geriátricos. En el caso de Fisioterapia, las preferencias fueron la atención primaria y pediatría, mientras que los enfermos terminales (de cáncer y geriátricos) resultaron las salidas menos deseadas. Los estudiantes de Medicina muestran sus preferencias con hospitales de agudos y grandes prematuros, siendo la opción de los enfermos terminales la menos elegida, al igual que los estudiantes de Fisioterapia.

Se observa que la alternativa de trabajo con enfermos terminales, ya sea enfermos de cáncer en fase terminal o geriátricos, es la opción menos aceptada por los futuros profesionales de la salud, al contrario que tratar a pacientes al inicio de la vida.

Una posible explicación a estos resultados puede relacionarse con el hecho de que el colectivo de la muestra, en mayor número, estaba formado por mujeres, y puede que el instinto maternal o la educación que han recibido desde la infancia sobre cuidar (reflejo de la figura materna) influyan en sus deseos laborales. Por otro lado, y como justificación a la poca aceptación del campo de trabajo con enfermos terminales, es que en él se refleja el tener que trabajar con la muerte, y el hecho de cuidar o de sentirse útil no están en primer término ${ }^{28,29}$. El alumno puede intuir que el sentirse útil con este tipo de pacientes (terminales) no es tan importante como en otras etapas vitales, donde el resultado de esos cuidados es la recompensa de la vida y la mejora en su calidad de vida $^{30}$. Ya Callahan, en el año $2000^{31}$, mostró que los objetivos de la Medicina (y afines) son dos, y con similar identidad: curar cuando se puede y, si no es posible, cuidar al enfermo.

Otra posibilidad a tener en cuenta es considerar que, por proximidad en la edad, se sientan más identificados con el hecho del nacimiento que con el de la muerte, pero esta afirmación es arriesgada, pues influye en estas cuestiones la experiencia vital de cada uno.
Una de las principales limitaciones del presente estudio está relacionada con el riesgo de sesgo en la selección de la muestra, ya que comprende un grupo de voluntarios y la falta de aleatorización. Al mismo tiempo, sería interesante poder realizar el mismo estudio en estudiantes de Ciencias de la Salud que ya hubiesen tenido un contacto más continuado con pacientes, para que pudieran expresar su opinión de manera más acorde con su experiencia vivida. Como propuesta para futuras investigaciones relacionadas, es necesario realizar trabajos que permitan ahondar más en este tema e identificar las necesidades formativas de los alumnos de Ciencias de la Salud en materia de final de la vida y muerte de los pacientes, junto con una muestra más amplia que permita extrapolar los resultados obtenidos. Igualmente, sería apropiado exponer los resultados obtenidos en este estudio a las distintas facultades de Ciencias de la Salud para que comprendan y valoren la implantación, desarrollo y profundización de sus estudiantes en el ámbito del cuidado de pacientes cercanos a la muerte.

En resumen, se observa poca predilección por los cuidados y tratamiento en el final de la vida. La preparación para poder atender a este tipo de pacientes por parte de los profesionales de la salud debe comenzar en la formación de grado, ya que los cambios de conducta y motivación hacia este campo de trabajo deben comenzar pronto (diseño adecuado de currículum formativo) y requieren de tiempo para su adquisición.

\section{Conflicto de intereses}

La autora declara no tener ningún conflicto de intereses.

\section{Bibliografía}

1. Aries P. Historia de la muerte en Occidente. Barcelona: El Acantilado; 2000.

2. Ascencio L, Allende S, Verastegui E. Creencias, actitudes y ansiedad ante la muerte en un equipo multidisciplinario de cuidados paliativos oncológicos. Psicooncología. 2014;11:101-15.

3. Sontag S. La enfermedad y sus metáforas. Buenos Aires: Taurus; 1996.

4. Gala FJ, Lupiani M, Díaz M. Sobre las concepciones de la muerte. Rol. 1991;159:63-6.

5. Benbunan-Bentata B, Cruz-Quintana F, Roa-Venegas JM, Villaverde-Gutiérrez C, Benbunan- Bentata BR. Afrontamiento del dolor y la muerte en estudiantes de enfermería: una propuesta de intervención. Int J Clin Health Psychol. 2007;7:197-205.

6. Gala FJ, Lupiani M, Raja R, Guillén C. Actitudes psicológicas ante la muerte y el duelo. Una revisión conceptual. Cuad Med Forense. 2002;30:39-50.

7. Corr CA. Death in modern society. En: Doyle D, Hanks GWC, MacDonald N, editores. Oxford texbook of palliative medicine. Oxford: Oxford Medical Publications; 1983; p. 28-36.

8. Tomás Sábado J, Guix Llistuella E. Ansiedad ante la muerte: Efectos de un curso de formación en enfermeras y auxiliares de enfermería. Enferm Clin. 2001;11:104-9.

9. Schmidt-Rio Valle J, Montoya-Juárez R, Campos-Calderón C, García-Caro MP, Prados-Peña D, Cruz-Quintana F. Efectos de un programa de formación en cuidados paliativos sobre el afrontamiento de la muerte. Med Paliat. 2012;19:113-20.

10. Carrasco JM, Lynch TJ, Garralda E, Woitha K, Elsner F, Filbet M, et al. Palliative care medical education in European Universi- 
ties: A descriptive study and numerical scoring system proposal for assessing educational development. J Pain Symptom Manage. 2015;50:516-23.

11. Mason S, Ellershaw J. Preparing for palliative medicine: Evaluation of an education programme for fourth year medical undergraduates. Palliat Med. 2008;22:687-92.

12. Vaquero J, Centeno C. Panorama actual de la enseñanza de medicina paliativa en la universidad española. Med Paliat. 2014;21:3-8.

13. Vallès $P$, García-Salvador I. Formación básica en cuidados paliativos: estado actual en las universidades de enfermería españolas. Med Paliat. 2013;20:111-4.

14. Parry M. Student nurses' experience of their first death in clinical practice. Int J Palliat Nurs. 2011;17:446-51.

15. Edo-Gual M, Tomás-Sábado J, Aradilla-Herrero A. Miedo a la muerte en estudiantes de enfermería. Enferm Clin. 2011;21:129-35.

16. Colell R. Análisis de las actitudes ante la muerte y el enfermo al final de la vida en estudiantes de enfermería de Andalucía y Cataluña [tesis doctoral]. Barcelona: Universidad Autónoma de Barcelona; 2005.

17. Allue Martínez M. ¿Cómo desearía morir, súbita o paulatinamente? Jano. 1988;34:82-93.

18. Bayés R, Limonero JT, Buendía E, Burón E, Enríquez N. Evaluación de la ansiedad frente a la muerte. Med Paliat. 1999;6:140-3.

19. Bayés R, Limonero JT, Arranz P. ¿Qué puede ayudarnos a morir en paz? Med Clin. 2000;115:579-82.

20. Centeno Cortés C, Núñez Olarte JM. Estudios sobre la comunicación del diagnóstico de cáncer en España. Med Clin. 1998; 110:744-50.

21. Esteban de la Rosa MA. El enfermo terminal y la muerte: Problemas médico-sociales, propuestas de información médica y organización sanitaria [tesis doctoral]. Murcia: Universidad de Murcia; 1995.
22. Rubio V, Sampedro E, Zapirain M, Gil I, Ayechu S, Tapiz, V. Diagnóstico: cáncer. ¿Queremos conocer la verdad? Aten Primaria. 2004;33:368-73.

23. Maté J, González J, Codorniú N, Barbero E. Abordaje emocional desde un modelo interdisciplinar en cuidados paliativos: A propósito de un caso. Libro de ponencias. III Congreso Nacional de la Sociedad Española de Psico-Oncología. 2007; p. 45.

24. Loncán P, Gisbert A, Fernández C, Valentín R, Teixidó A, Vidaurreta $\mathrm{R}$, et al. Cuidados paliativos y medicina intensiva en la atención al final de la vida del siglo XXI. An Sist Sanit Navar. 2007;30:113-28.

25. Gray B. The emotional labour of nursing defining and managing emotions in nursing work. Nurs Educ Today. 2009;29:168-75.

26. Rojas Alcántara P, Armero Barranco D, Campos Aranda G, Muñoz Pérez VM, Riquelme Martín A, González Quijano A. Actitud de los jóvenes universitarios en ciencias de la salud ante el dolor y el sufrimiento en el proceso de muerte. Enfermería Científica. 2004;265:61-8.

27. Colell Brunet R, Limonero García JT, Otero MD. Actitudes y emociones en estudiantes de enfermería ante la muerte y la enfermedad terminal. Investigación en Salud [Internet]. 2003 Agosto [cited: 2016 Jun 12];5(2):[about 10 p.] Disponible en: http: / / www.redalyc.org/articulo.oa?id=14250205.

28. González Cervantes S. Actitudes ante la muerte en estudiantes de grado en Enfermería [tesis doctoral]. Madrid: Universidad Complutense de Madrid; 2015.

29. Camps V. La excelencia en las profesiones sanitarias. Humanitas Humanidades Médicas. 2007;1:1-13.

30. Collel R, Fontanals A, Jürschik P, Torner T, Allende P. La relación con el enfermo terminal. Una difícil realidad. Notas de Enfermería. 1991;1:19-22.

31. Callahan D. Death and the research imperative. N Engl J Med. 2000;342:654-6. 